\title{
Effect of dexmedetomidine infusion on N-terminal pro-B-type natriuretic peptide level in patients with femoral shaft fractures under general anesthesia
}

\author{
Alireza Mirkheshti, Elham Memary, Hamed Shafiee and Mastaneh Dahi \\ Department of Anesthesiology, Imam Hossein Hospital, Shahid Beheshti University of Medical Sciences, Tehran, \\ Iran.
}

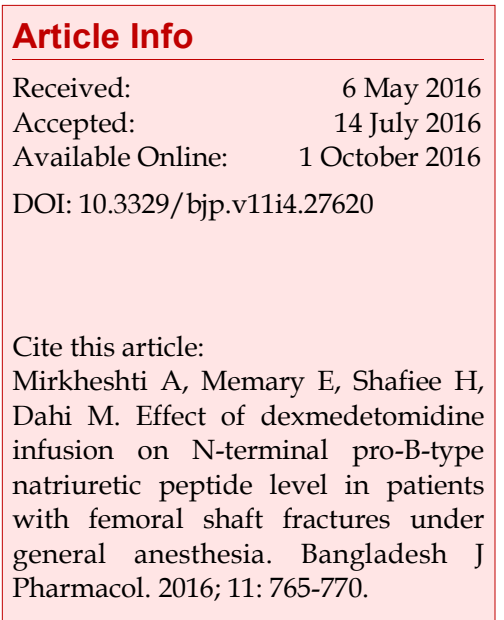

\begin{abstract}
This study was performed to assess the effect of dexmedetomidine on the level of N-terminal pro-B-type natriuretic peptide (NT-pro-BNP) in patients undergoing major orthopedic surgery. Patients undergoing general anesthesia for femoral shaft fracture surgery were randomly assigned to either $0.5 \mu \mathrm{g} /$ $\mathrm{kg} /$ hour dexmedetomidine or normal saline. Changes in levels of NT-proBNP and hemodynamic parameters were compared. Data of 46 patients were analyzed and it was found that the change in NT-pro-BNP levels in the dexmedetomidine group was significantly less than the control group $(p=$ 0.001). In addition, bleeding, changes in systolic and diastolic blood pressure in dexmedetomidine group was significantly less than the control group $(\mathrm{p}<0.001)$. It is likely that dexmedetomidine infusion can reduce the rise of NT -pro-BNP level and therefore is associated with better cardiac outcome. In addition, dexmedetomidine infusion can achieve hemodynamic stability in femoral shaft fracture under general anesthesia.
\end{abstract}

\section{Introduction}

Brain natriuretic peptide is a 32-amino acid peptide whose plasma levels or its N-terminal fragment (NTpro-BNP) level are being increasingly applied for cardiac assay in perioperative settings. The literature suggests that brain natriuretic peptide $<20 \mathrm{pg} / \mathrm{mL}$ and NT-pro-BNP $<125 \mathrm{pg} / \mathrm{mL}$ are appropriate cut points to rule out heart failure in asymptomatic patients (Betti et al., 2009; Chong et al., 2010; Tepper et al., 2009). Agents affecting these levels in the plasma will help monitor and manage cardiac functions.

The a-2 receptor agonists are used preoperatively as they have many desirable effects such as analgesia, inhibition of sympathetic outflow, anxiolytics, and reduction of norepinephrine release, which improve hemodynamic stability and have positive effects on myocardial oxygen supply and demand, and may even offer myocardial protection (Ellis et al., 1994; Muzi et al., 1992).

Dexmedetomidine is a highly selective, intravenous $a-2$ agonist and has an a-2 to a-1 selectivity ratio of 1600:1 (Khan et al., 1999). Perianesthetic use of dexmedetomidine has a hemodynamic stability effect, which is associated with a decrease in postoperative mortality and reduction in the incidence of postoperative complications (Ji et al., 2013; Shin et al., 2013). The protective effects of dexmedetomidine on postoperative myocardial injury have been defined (Ren et al., 2013).

The purpose of this randomized controlled trial was to investigate the effect of dexmedetomidine on the level of NT pro-B-type natriuretic peptide in patients undergoing major orthopedic surgery and to investigate the effects of dexmedetomidine infusion on intraoperative bleeding and hemodynamic status of these patients. 


\section{Materials and Methods}

\section{Study design}

We designed a randomized controlled trial in Imam Hossein Hospital, Tehran, Iran from August 2014 until September 2015.

\section{Study population}

Patients with femoral shaft fracture between 15 and 55 years, American Society of Anesthesiologists (ASA) Class I or II, who were the candidate for elective surgery under general anesthesia were randomly recruited to our study using randomized numbers table.

\section{Exclusion criteria}

We excluded patients in the following conditions: a) history of any known cardiac or hepatic disease; b) drug addiction; c) pregnancy; d) patients under treatment with antihypertensive, psychiatric, agonist or antagonist a-2 receptor agents; e) patients with basal heart rate less than $60 / \mathrm{min}$; f) patients with blood loss of more than 1 liter during surgery; g) patients for whom the time of operation took more than 3 hours.

\section{Induction of anesthesia}

All patients received $20 \mathrm{~mL} / \mathrm{kg}$ normal saline, $2 \mu \mathrm{g} / \mathrm{kg}$ fentanyl, and $0.02 \mathrm{mg} / \mathrm{kg}$ midazolam intravenously as premedication. Induction of anesthesia was performed intravenously by $2 \mathrm{mg} / \mathrm{kg}$ propofol and $0.5 \mathrm{mg} / \mathrm{kg}$ atracurium. Anesthesia was maintained by $150 \mu \mathrm{g} / \mathrm{kg} /$ min propofol and $\mathrm{O} 2 / \mathrm{N} 2 \mathrm{O}$ as 2/2 liters.

\section{Intervention}

In the DEX group (named as D), the patients received the infusion of dexmedetomidine hydrochloride (Precedex $200 \mathrm{mg} / 2 \mathrm{~mL}$, USA) as $0.5 \mu \mathrm{g} / \mathrm{kg} /$ hour diluted in normal saline and in the control group (named as C), the same volume of normal saline was infused.

\section{Monitoring of patient case and pain management}

Patients were monitored by continuous evaluation of their heart rates, blood pressure, oxygen saturation, end tidal CO2, and electrocardiograms. The protocol of postoperative pain management through an infusion pump of morphine as intravenous patient-controlled analgesia by lockout time of $10 \mathrm{~min}$ was observed in all patients during 24 hours.

\section{Primary outcome measures}

Change in the levels of NT-pro-BNP

The change in NT-pro-BNP was calculated from two blood samples (preoperative and postoperative) obtained from each patient. The first blood sample was taken in the operation room using an 18 gauge catheter.
While the second sample of NT-pro-BNP was taken 24 hours after the operation.

Amount of intraoperative bleeding

The intraoperative bleeding was estimated based on the volume of blood in the suction bottle and the weight of the bloody gauze pads. Every pack of the surgical pad was weighed pre- and post-blood soaking and by considering each milliliter of blood nearly equal to 1.06 $\mathrm{mg}$. The final blood loss was estimated by weighing the pads and adding it to the volume of blood in the suction bottle.

\section{Secondary outcome measures}

Secondary outcomes include a) the change in the systolic and the diastolic blood pressure in both groups and $b$ ) the incidence of cardiovascular adverse events and high-risk cardiac state.

\section{Sample size calculation}

We calculated the sample size assuming 5\% margin of error and $80 \%$ power to detect a change in BNP level of 70 with SD $=85$ in each group. A sample of 24 patients in each group was needed. One patient in the DEX group was excluded because the time of operation exceeded more than 3 hours.

\section{Statistical analysis}

The Kolmogorov-Smirnov test and Q-Q plot were used to examine the normal distribution of data. To describe data, we used mean, standard deviation, median and inter-quartile range, frequency, and percentage. To compare the results between the two groups, we used the t-test, Mann-Whitney test, and chi-square test. To compare the results after adjusting the baseline values, we used Analysis of Covariance (ANCOVA). To compare the trend of change in SBP, DBP, and PR between the two groups, we used linear mixed model analysis. All statistical analyses were performed by SPSS (IBM SPSS for Windows, Version 22.0. Armonk, NY: IBM Corp.). An alpha level below 0.05 was considered statistically significant.

\section{Results}

Two patients in each group were excluded and finally, the 46 patient's data were analyzed (Figure 1). Of the 50 patients recruited in the trial, two patients in each group were excluded from the final analysis because of excess blood loss and long lasting the surgery. Demographic and baseline characteristics of the study population are shown in Table I.

Changes in NT-pro-BNP levels were less in the group D than group $C(p=0.001)$. In addition, the amount of bleeding in the group $\mathrm{D}$ was less than group $\mathrm{C}$ $(\mathrm{p}<0.001$; Table II $)$ 
Table I

Demographic data

\begin{tabular}{|c|c|c|c|c|c|}
\hline \multirow[t]{2}{*}{ Parameter } & & \multirow[t]{2}{*}{ Total } & \multicolumn{2}{|c|}{ Group } & \multirow[t]{2}{*}{$\mathrm{p}$} \\
\hline & & & Dexmedetomidine & Placebo & \\
\hline \multirow[t]{2}{*}{ Age (years) } & Mean \pm SD & $41 \pm 14$ & $43 \pm 13$ & $40 \pm 16$ & $0.507 \dagger$ \\
\hline & Median (IQR) & 40 (31 to 51$)$ & 48 (31 to 55$)$ & 35 (31 to 49$)$ & \\
\hline \multirow[t]{2}{*}{ Gender } & M & $33(71.7 \%)$ & $15(65.2 \%)$ & $18(78.3 \%)$ & $0.464^{*}$ \\
\hline & $\mathrm{F}$ & $13(28.3 \%)$ & $8(34.8 \%)$ & $5(21.7 \%)$ & \\
\hline \multirow[t]{2}{*}{ Weight $(\mathrm{kg})$} & Mean \pm SD & $69 \pm 10$ & $69 \pm 12$ & $69 \pm 7$ & $0.874 \dagger$ \\
\hline & Median (IQR) & 70 (65 to 75$)$ & $67(60$ to 80$)$ & 70 (67 to 73$)$ & \\
\hline \multirow[t]{2}{*}{ BNPPRE } & Mean \pm SD & $36 \pm 28$ & $40 \pm 37$ & $33 \pm 17$ & $0.773 \ddagger$ \\
\hline & Median (IQR) & 20 (20 to 45$)$ & 20 (20 to 55$)$ & 26 (20 to 43$)$ & \\
\hline \multirow[t]{2}{*}{ SBP0 } & Mean \pm SD & $100 \pm 12$ & $10113 \pm$ & $99 \pm 10$ & $0.570 \dagger$ \\
\hline & Median (IQR) & 100 (95 to 102$)$ & 102 (97 to 112 ) & 100 (95 to 100$)$ & \\
\hline \multirow[t]{2}{*}{ DBP0 } & Mean \pm SD & $67 \pm 10$ & $67 \pm 11$ & $67 \pm 8$ & $0.770 \dagger$ \\
\hline & Median (IQR) & 68 (62 to 74$)$ & 67 (58 to 78$)$ & $70(65$ to 70$)$ & \\
\hline \multirow[t]{2}{*}{ PR0 } & Mean \pm SD & $89 \pm 16$ & $85 \pm 12$ & $92 \pm 18$ & $0.143 \dagger$ \\
\hline & Median (IQR) & 85 (75 to 100$)$ & 85 (75 to 95$)$ & 90 (80 to 100$)$ & \\
\hline
\end{tabular}

†Based on t-test; ‡Based on Mann-Whitney test; *Based on Chi-Square test

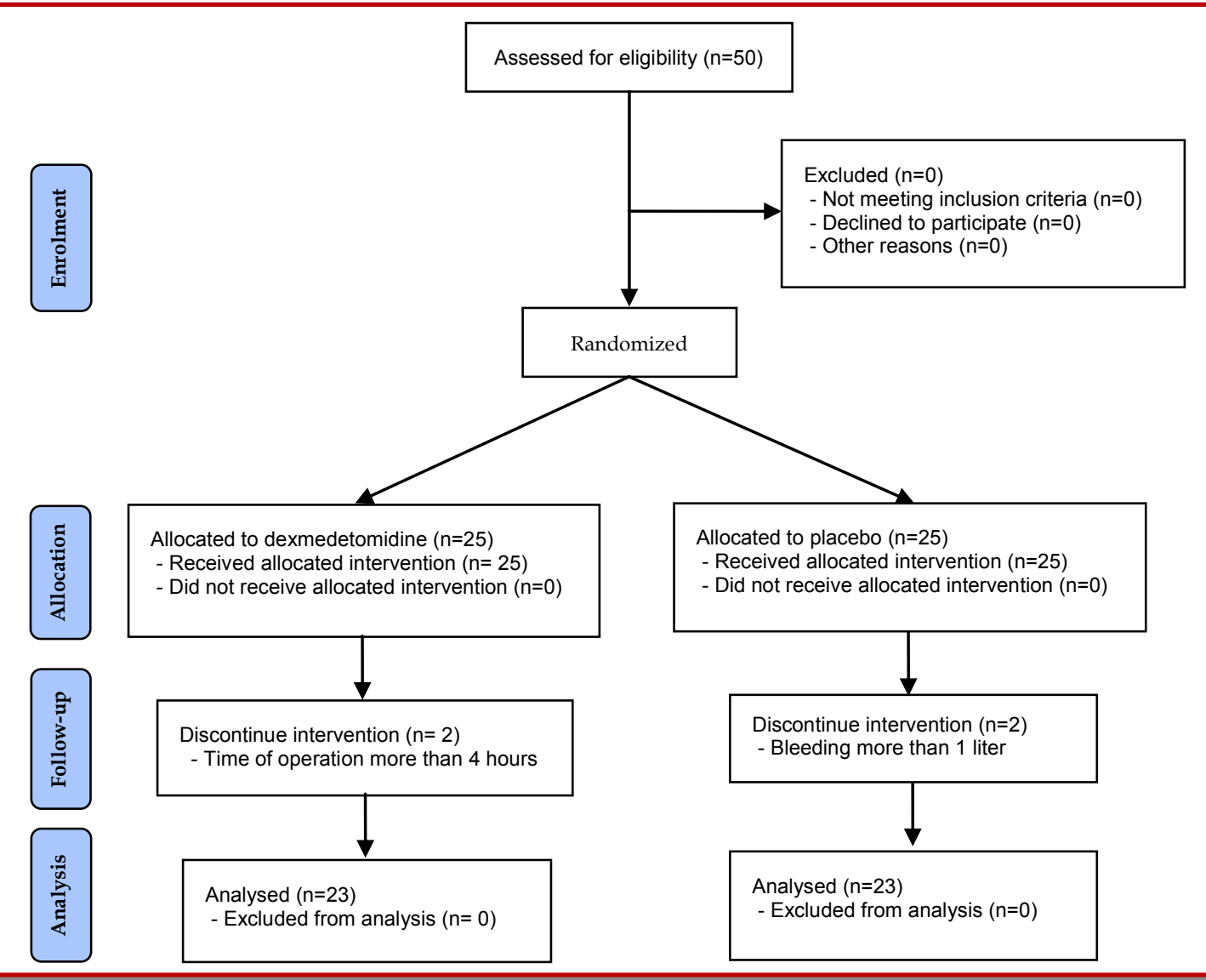

Figure 1: Consort diagram showing the flow of participants 
Table II

Changes in N-terminal pro-B-type natriuretic peptide and bleeding volume

\begin{tabular}{|c|c|c|c|c|c|c|c|}
\hline \multirow[t]{2}{*}{ Parameter } & & \multicolumn{2}{|c|}{ Group } & \multirow[t]{2}{*}{ Difference } & \multicolumn{2}{|c|}{$95 \% \mathrm{CI}$} & \multirow[t]{2}{*}{$\mathrm{p}$} \\
\hline & & Dexmedetomidine & Placebo & & Lower & Upper & \\
\hline \multirow[t]{2}{*}{ NT pro-BNP POST } & Mean \pm SD & $55 \pm 96$ & $132 \pm 95$ & -76.5 & -132.7 & -20.4 & $<0.001 ¥$ \\
\hline & Median (IQR) & 20 (20 to 65$)$ & 104 (70 to 174$)$ & & & & \\
\hline \multirow[t]{2}{*}{ NT pro-BNP change } & Mean \pm SD & $15 \pm 67$ & $99 \pm 96$ & -84.1 & -133.0 & -35.3 & $0.001 \ddagger$ \\
\hline & Median (IQR) & $0(0$ to 8$)$ & 75 (38 to 145$)$ & & & & \\
\hline \multirow[t]{2}{*}{ Bleeding $(\mathrm{mL})$} & Mean \pm SD & $440 \pm 115$ & $620 \pm 178$ & -179.1 & -267.6 & -90.7 & $<0.001 \dagger$ \\
\hline & Median (IQR) & 440 (350 to 500$)$ & $600(465$ to 725$)$ & & & & \\
\hline
\end{tabular}

†Based on t-test; ‡Based on Mann-Whitney test; ¥Adjusted for the baseline value based on Analysis of Covariance (ANCOVA)

The mixed model analysis showed a statistically significant difference in the systolic blood pressure between the dexmedetomidine and the control groups $(p<0.001$; Figure 2). In addition, the analysis of trend showed a statistically significant change in diastolic blood pressure between D and C groups $(p<0.001$; Figure 2). Although both groups showed a significant decrease in pulse rate during the surgery $(p<0.001)$ but there was no trend for a statistically significant difference between two group ( $p=0.055$; Figure 3$)$.

There were no postoperative cardiac complications as heart failure, arrhythmia, or ischemic events in either of the groups. Based on BNP cut point, only one patient was at risk of postoperative cardiac events in the $D$ group and none of the $\mathrm{C}$ group was at risk of such complications. After the operation, no new cases developed high-risk cardiac state in the $\mathrm{D}$ group but 11 patients $(47 \%)$ of the $C$ group developed the high-risk cardiac state. This difference between the two groups was statistically significant as indicated by chi-square test $(\mathrm{p}=0.001)$. The odds of high cardiac state after operation in the D group were 20.2 (95\% CI 2.3 to 175.7) times higher than the $\mathrm{C}$ group.

\section{Discussion}

Our results suggest that dexmedetomidine significantly affects the levels of NT-pro-BNP level following femoral shaft fracture surgery under general anesthesia. The levels of NT-pro-BNP in the blood is a reliable predictor of postoperative cardiac events within 30 days after non -cardiac surgery and dexmedetomidine significantly decreased the risk of developing high-risk cardiac state up to 20 -fold in cases with an ASA class I or II.

The rise of NT-pro-BNP in major orthopedic surgery is in agreement with the study of Vetrugno et al. in which they evaluated the change in NT-pro-BNP as a predictor of in-hospital major adverse cardiac events
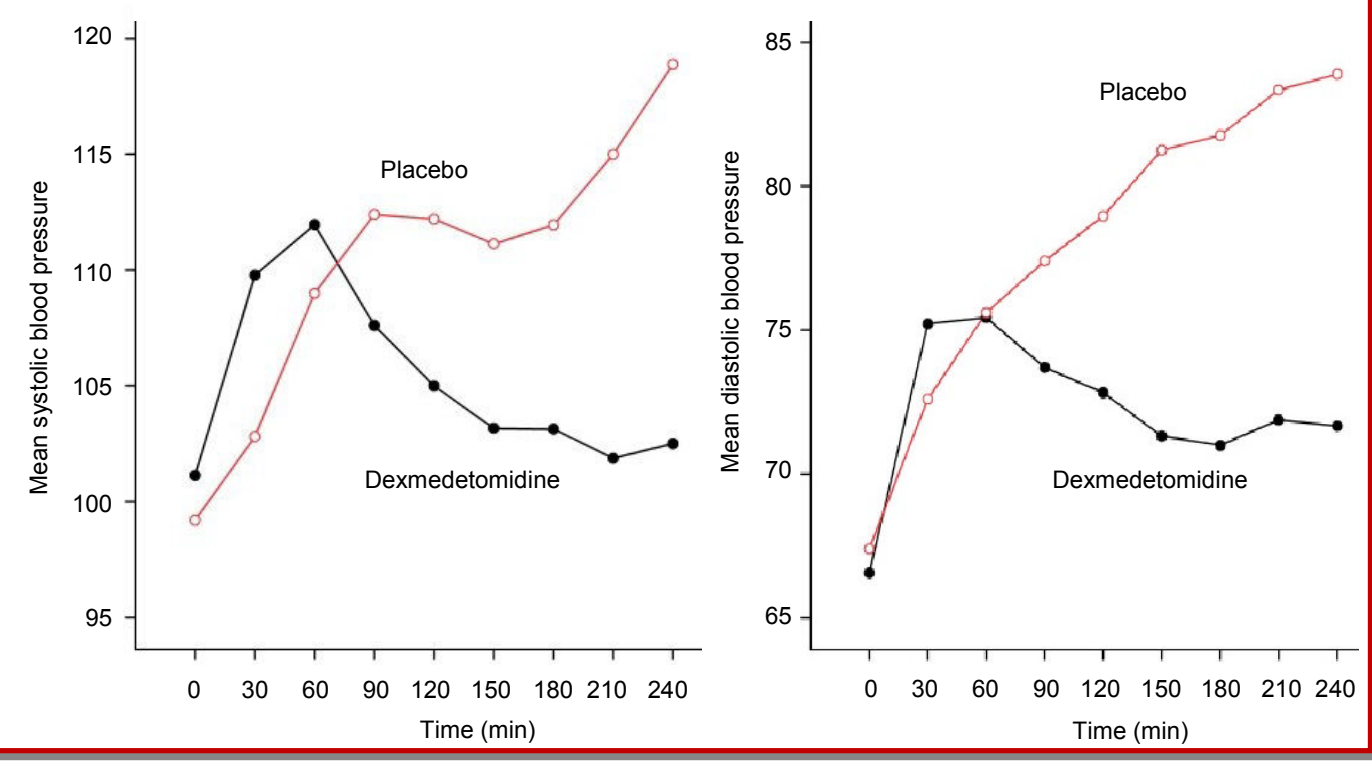

Figure 2: Mean systolic and diastolic blood pressure changes 


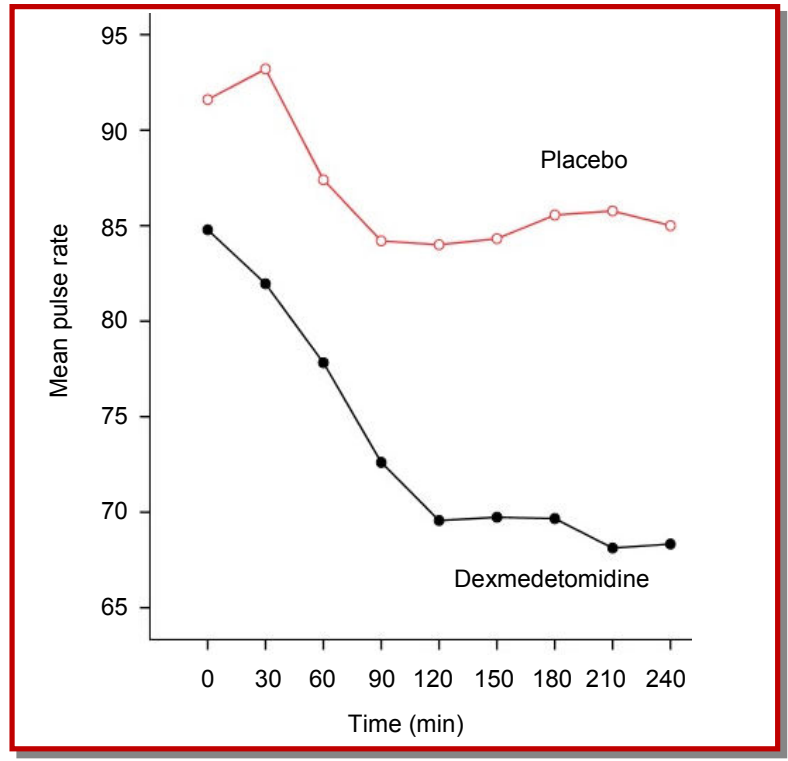

Figure 3: Mean pulse rate changes

(MACE: Atrial fibrillation, flutter, acute heart failure, or non-fatal/fatal myocardial infarction) in patients undergoing elective prosthesis orthopedic surgery. They found that both pre and postoperative BNP concentrations are predictors of MACE in patients undergoing elective prosthesis orthopedic surgery (Vetrugno et al., 2014). In our study, we showed that in the D group, the rise of NT-pro-BNP was less than in the control group, which is in agreement with the survey (Biccard et al., 2008). Mizrak et al. evaluated the effect of DEX on intraoperative blood loss in pediatric adenotonsillectomy and they concluded that dexmedetomidine slightly increased bleeding during the operation (Mizrak et al., 2013). These results are different from our findings because we evaluated the effect of dexmedetomidine infusion during operation, but in Mizrak et al, (2013) study, they evaluated only a bolus dose of the drug as premedication. In the systematic review of Biccard et al. they evaluated the effects of dexmedetomidine on cardiac outcomes after non-cardiac surgery. They found that dexmedetomidine was associated with better cardiac outcomes which are concordant with our findings (Biccard et al., 2008). In terms of controlling blood loss, our results are in agreement with the study by Ibraheim et al., where they found dexmedetomidine an effective method to reduce the amount of bleeding in patients undergoing scoliosis surgery (Ibraheim et al., 2013). In terms of controlling the hemodynamic parameters, our findings are consistent with the survey of Mariappan et al, (2014) where they found dexmedetomidine to be effective in controlling the hemodynamic response and reducing the blood loss during spine surgery (Mariappan et al., 2014). It was not possible to measure NT-pro-BNP levels in shorter intervals, and we excluded patients with known heart disease and ASA class $\amalg$ or greater.

\section{Conclusion}

Dexmedetomidine infusion can reduce the rise of NTpro-BNP level and therefore is associated with better cardiac outcome.

\section{Acknowledgement}

We would like to thank all anesthesiology department staff of Imam Hossein Hospital who helped in management of patients.

\section{Ethical Issue}

The protocol of this study was approved by the Ethics Committee of Shahid Beheshti University of Medical Sciences, Iran. Patients were informed about the study details and they signed a written consent. All authors declare to the Helsinki in ethical principles for medical research involving human subjects throughout the study. We followed the CONSORT statement guidelines during the preparation and reporting of this randomized controlled trials (Moher et al., 2010). This methodology of this trial was registered on www.IRCT.IR with the registration number IRCT201511279593N2.

\section{Conflict of Interest}

All authors have completed the ICMJE uniform disclosure form and declare no support from any organization for the submitted work.

\section{References}

Betti I, Castelli G, Barchielli A, Beligni C, Boscherini V, De Luca L, Messeri G, Gheorghiade M, Maisel A, Zuppiroli A. The role of N-terminal PRO-brain natriuretic peptide and echocardiography for screening asymptomatic left ventricular dysfunction in a population at high risk for heart failure. The PROBE-HF study. J Card Fail. 2009; 15: 377-84.

Biccard B, Goga S, De Beurs J. Dexmedetomidine and cardiac protection for non-cardiac surgery: A meta-analysis of randomised controlled trials. Anaesthesia 2008; 63: 4-14.

Chong CP, Ryan JE, van Gaal WJ, Lam QT, Sinnappu RN, Burrell LM, Savige J, Lim WK. Usefulness of N-terminal probrain natriuretic peptide to predict postoperative cardiac complications and long-term mortality after emergency lower limb orthopedic surgery. Am J Cardiol. 2010; 106: 86572 .

Ellis JE, Drijvers G, Pedlow S, Laff SP, Sorrentino MJ, Foss JF, Shah M, Busse JR, Mantha S, McKinsey JF, et al. Premedication with oral and transdermal clonidine provides safe and efficacious postoperative sympatholysis. Anesth Analg. 1994; 79: 1133-40.

Ibraheim OA, Abdulmonem A, Baaj J, Zahrani T, Arlet V. Esmolol versus dexmedetomidine in scoliosis surgery: Study on intraoperative blood loss and hemodynamic changes. 
Middle East J Anesthesiol. 2013; 22: 27-33.

Ji F, Li Z, Nguyen H, Young N, Shi P, Fleming N, Liu H. Perioperative dexmedetomidine improves outcomes of cardiac surgery. Circulation 2013; 112: 000936.

Khan Z, Ferguson C, Jones R. Alpha-2 and imidazoline receptor agonists their pharmacology and therapeutic role. Anaesthesia 1999; 54: 146-65.

Mariappan R, Ashokkumar H, Kuppuswamy B. Comparing the effects of oral clonidine premedication with intraoperative dexmedetomidine infusion on anesthetic requirement and recovery from anesthesia in patients undergoing major spine surgery. J Neurosurg Anesthesiol. 2014; 26: 192-97.

Mizrak A, Karatas E, Saruhan R, Kara F, Oner U, Saricicek V, Baysal E. Does dexmedetomidine affect intraoperative blood loss and clotting tests in pediatric adenotonsillectomy patients? J Surg Res. 2013; 179: 94-98.

Moher D, Hopewell S, Schulz KF, Montori V, Gotzsche PC, Devereaux PJ, Elbourne D, Egger M, Altman DG. CONSORT 2010 explanation and elaboration: Updated guidelines for reporting parallel group randomised trials. J Clin Epidemiol. 2010; 63: e1-37.
Muzi M, Goff DR, Kampine JP, Roerig DL, Ebert TJ. Clonidine reduces sympathetic activity but maintains baroreflex responses in normotensive humans. Anesthesiology 1992; 77: 864-71.

Ren J, Zhang H, Huang L, Liu Y, Liu F, Dong Z. Protective effect of dexmedetomidine in coronary artery bypass grafting surgery. Exp Ther Med. 2013; 6: 497-502.

Shin HW, Yoo HN, Kim DH, Lee H, Shin HJ, Lee HW. Preanesthetic dexmedetomidine $1 \mu \mathrm{g} / \mathrm{kg}$ single infusion is a simple, easy, and economic adjuvant for general anesthesia. Korean J Anesthesiol. 2013; 65: 114-20.

Tepper D, Harris S, Ip R. The role of N-terminal pro-brain natriuretic peptide and echocardiography for screening asymptomatic left ventricular dysfunction in a population at high-risk for heart failure: The PROBE-HF Study. Congestive Heart Failure. 2009; 15: 296.

Vetrugno L, Langiano N, Gisonni R, Rizzardo A, Venchiarutti PE, Divella M, Della Rocca G. Prediction of early postoperative major cardiac events after elective orthopedic surgery: The role of B-type natriuretic peptide, the revised cardiac risk index, and ASA class. BMC Anesthesiol. 2014; 14: 1 . 


\section{Your feedback about this paper}

1. Number of times you have read this paper 0

2. Quality of paper Click

3. Your comments

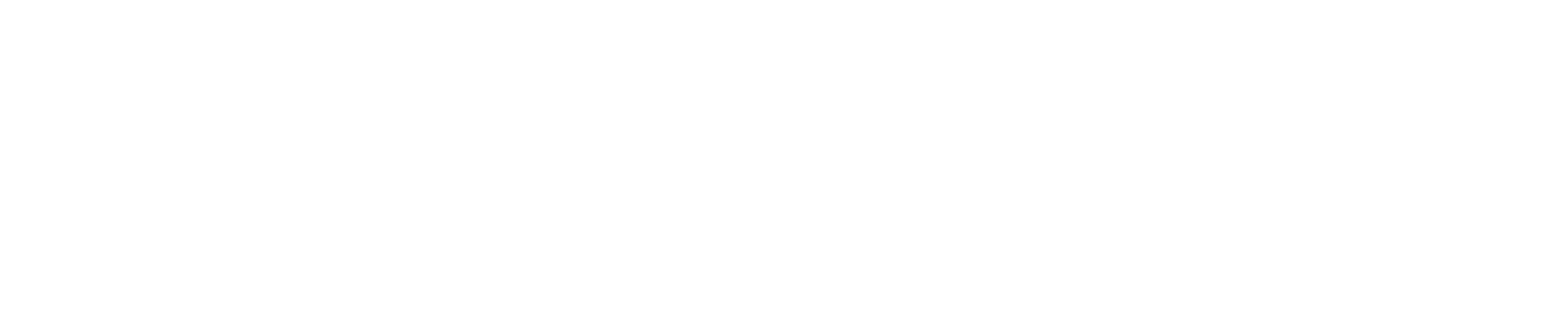

